\title{
A Solution to the Cosmological Constant Problem in Two Time Dimensions
}

\author{
Christoph Köhn \\ Technical University of Denmark, National Space Institute (DTU Space), Kgs Lyngby, Denmark \\ Email:koehn@space.dtu.dk
}

How to cite this paper: Köhn, C. (2020) A Solution to the Cosmological Constant Problem in Two Time Dimensions. Journal of High Energy Physics, Gravitation and Cosmology, 6, 640-655.

https://doi.org/10.4236/jhepgc.2020.64043

Received: August 10, 2020

Accepted: September 6, 2020

Published: September 9, 2020

Copyright $\odot 2020$ by author(s) and Scientific Research Publishing Inc. This work is licensed under the Creative Commons Attribution International License (CC BY 4.0).

http://creativecommons.org/licenses/by/4.0/

\begin{abstract}
For the last hundred years, the existence and the value of the cosmological constant $\Lambda$ has been a great enigma. So far, any theoretical model has failed to predict the value of $\Lambda$ by several orders of magnitude. We here offer a solution to the cosmological constant problem by extending the Einstein-Friedmann equations by one additional time dimension. Solving these equations, we find that the Universe is flat on a global scale and that the cosmological constant lies between $10^{-90} \mathrm{~m}^{-2}$ and $10^{-51} \mathrm{~m}^{-2}$ which is in range observed by experiments. It also proposes a mean to explain the Planck length and to mitigate the singularity at the Big Bang.
\end{abstract}

\section{Keywords}

Two Time, Dimensions, Planck Length, Cosmological Constant Problem, Curvature of the Universe

\section{Introduction}

The history of the cosmological constant has been inferred with ups and downs. Originally, it was introduced by Einstein [1] to explain a steady-state Universe. When it was, however, discovered that the size of the Universe is not steady, Einstein discarded his idea of the cosmological constant and called it "die größte Eselei meines Lebens" (the biggest blunder of my life).

Since the early 1990s, it has been known that the energy-matter content of the Universe consists not only of visible baryonic matter which contributes $\approx 5 \%$ to the Universe's energy, but also of Dark Matter $(\approx 23 \%)$ and a phenomenon called Dark Energy $(\approx 72 \%)$ which is related to the expansion of the Universe and probably associated to the cosmological constant [2]-[7] where measurements of the Universe's expansion suggest a value of approximately $10^{-52} \mathrm{~m}^{-2}$ [8] [9] [10] [11]. In current theories, the cosmological constant has a fundamental meaning for certain phenomena in our Universe, such as on the clustering of galaxies [12] 
or on the gravity's rainbow effect [13] of black holes on the propagation of photons with different wave lengths where the cosmological constant is treated equivalent to the thermodynamic pressure [14] [15]. This latter property of the cosmological constant is, for example, also used to derive a relation amongst geometry, thermodynamics and information theory by using complexity [16].

There have been a plethora of attempts to derive the value of the cosmological constant theoretically. However, such calculations yield predictions which are many orders of magnitude larger than $\approx 10^{-52} \mathrm{~m}^{-2}$ which is also known as the "cosmological constant problem". Zel'dovich related all elementary particles and the quantum fluctuations in the Universe to the background energy which manifests as the Dark Energy in General Relativity. By this approach, he, however, found a value of the cosmological constant which is approximately 20 orders of magnitude larger than the measured value [17] [18]; other authors quantize these discrepancy to even 120 orders of magnitude [19] [20]. Another approach to explain Dark Energy are Extended Theories of Gravity where Dark Energy does not result from the cosmological constant and thus makes it obsolete, but becomes manifest from higher order curvature terms which are added to the standard Einstein-Hilbert action, see [21] and references therein. More recently, Marcolli and Perpaoli coupled gravity to matter with methods of noncommutative geometry and found a variable cosmological constant. They give an analytic solution for the effective cosmological constant with values of $10^{60} \mathrm{~m}^{-2}$, again larger than the measured value [22]. Garattini, Kruglov and Faizal showed that the cosmological constant might originate from a deformation of the Wheeler-DeWitt equation [23] [24]. Furthermore, this approach shows that the existence of the cosmological constant might exclude the Big Bang singularity.

In 1996 Bars showed that a certain class of string theories contains more than one time dimension [25]. This idea was further developed by Bars and Kounnas [26] [27] constructing actions for interacting p-branes within two dimensions. This yields a phase transition where the additional time dimension becomes part of the compactified universe. Additionally, they present a new Kaluza-Klein like dimensional reduction mechanism together with an action for a string in two time dimensions. Based on such a second time dimension, Araya and Bars showed that the Lagrangians and Hamiltonians of one-time systems which do not appear to be connected to each other, can be dualised by introducing a second time dimensions [28]. As an example, they show that the Lagrangians of massive relativistic and massless relativistic or massless relativistic and massive non-relativistic systems are dual to each other through such hidden relations.

Chen [29] later interpreted two extra time dimensions as hidden quantum variables where the non-local properties of quantum physics or the idea of matter waves using the de Broglie wave length are natural consequences from the action of a free particle in several time dimensions. Combining two-time physics with noncommutative $S p(2, R)$ gauge theories leads to theories containing all known results of two-time physics including the reduction of physical spacetime with the associated "holography" and "duality" properties [30]. Based on such an 
$\operatorname{Sp}(2, R)$ gauge symmetry, the Becchi-Rouet-Stora-Tyutin (BRST) formulation then allows to construct a field theoretic formulation of two-time physics including interactions. Such a scenario determines the interaction at the level of the action uniquely by the interacting BRST gauge symmetry and opens a way to study two-time physics on the quantum level through the path integral approach [31]. Based on Romero's and Zamora's work on the relation between two-time physics and the Snyder noncommutative Space [32], Carrisi and Mignemi extended this model to seven dimensions deriving the symplectic structure of the Snyder model on a de-Sitter background [33].

Although theoretically possible, causality puts certain constraints on the properties of time dimensions summarized by Tegmark [34]. In the case of three spatial dimensions, more than one macroscopic time dimension would lead to an unpredictable Universe. Therefore, any additional time dimension must be small or at least be irrelevant for the macroscopic observables of the Universe. However, even in this case, a second time dimension puts certain constraints on macroscopic Newtonian and quantum physics: Whilst a second time dimension puts strong constraints on the connections coming from gauge interactions in Newtonian physics, it leads to a generalized uncertainty relation involving level spacings and Planck's constant on the quantum level [35].

In previous work [36], we coupled the Lagrangian of a free, relativistic particle to a second time dimension, small enough to not violate the causality of the observable Universe. Starting from this Lagrangian, we showed that a small length scale, the Planck length, emerged from this theory and that the speed of light was not constant in the Early Universe, as predicted by Albrecht and Magueijo [37].

We now proceed one step further and extend the Robertson-Walker-LemaitreFriedmann metric by one additional time dimension.

\section{The Friedmann Equations in Five-Dimensional Space-Time}

Our starting point is the extension of the four-vector

$$
x^{\mu}=\left(\begin{array}{c}
t \\
r \\
\theta \\
\varphi
\end{array}\right) \rightarrow x^{\mu}=\left(\begin{array}{c}
t \\
\tau \\
r \\
\theta \\
\varphi
\end{array}\right)
$$

to a five-vector with a second time dimension $\tau$ expanding four-dimensional space-time with time dimension $t$ to five-dimensional space-time. Similarly, we extend the Robertson-Walker-Lemaitre-Friedmann metric [38] [39] from

$$
g_{\mu \nu}=\left(\begin{array}{cccc}
c(t)^{2} & 0 & 0 & 0 \\
0 & \frac{-a(t)^{2}}{1-\frac{r^{2}}{K}} & 0 & 0 \\
0 & 0 & -a(t)^{2} \cdot r^{2} & 0 \\
0 & 0 & 0 & -a(t)^{2} \cdot r^{2} \cdot \sin (\theta)^{2}
\end{array}\right)
$$


to

$$
\rightarrow g_{\mu \nu}=\left(\begin{array}{ccccc}
c(t)^{2} & 0 & 0 & 0 & 0 \\
0 & f(\tau)^{2} & 0 & 0 & 0 \\
0 & 0 & \frac{-a(t, \tau)^{2}}{1-\frac{r^{2}}{K}} & 0 & 0 \\
0 & 0 & 0 & -a(t, \tau)^{2} \cdot r^{2} & 0 \\
0 & 0 & 0 & 0 & -a(t, \tau)^{2} \cdot r^{2} \cdot \sin (\theta)^{2}
\end{array}\right)
$$

where $a$ is the scaling parameter of the Universe, $|K|$ is the inverse square of the curvature and $f$ the characteristic speed for the second time dimension such as $c$ for the first time dimension [36]. Here, $K$ is chosen such that the Universe is hyperbolic for $\operatorname{sign}(K)<0$, parabolic for $\operatorname{sign}(K)>0$ and flat and steady for $1 / K \equiv 0$. Note that, instead of using the conventional definition $x^{\mu}=(c t, f \tau, r, \theta, \varphi)$, we have included the speeds $c$ and $f$ in the five-dimensional metric. For full generality, we start with time depending velocities $c$ and $f$ as proposed for the early Universe [36] [37].

From metric (3), we derive the Christoffel symbols and subsequently the Ricci tensor $R_{\mu v}$ and the Ricci scalar $R$ (which are summarized in the supplementary Mathematica script).

In order to formulate Einstein's field equations, we also extend the energy-momentum tensor

$$
\begin{aligned}
& T_{\mu v}=\left(\begin{array}{cccc}
c(t)^{2} \rho(t) & 0 & 0 & 0 \\
0 & -p(t) g_{11} & 0 & 0 \\
0 & 0 & -p(t) g_{22} & 0 \\
0 & 0 & 0 & -p(t) g_{33}
\end{array}\right) \\
& \rightarrow\left(\begin{array}{ccccc}
c(t)^{2} \rho(t, \tau) & c(t) f(\tau) \rho(t, \tau) & 0 & 0 & 0 \\
c(t) f(\tau) \rho(t, \tau) & f(\tau)^{2} \rho(t, \tau) & 0 & 0 & 0 \\
0 & 0 & -p(t, \tau) g_{33} & 0 & 0 \\
0 & 0 & 0 & -p(t, \tau) g_{44} & 0 \\
0 & 0 & 0 & 0 & -p(t, \tau) g_{55}
\end{array}\right)
\end{aligned}
$$

with the density $\rho$ and the pressure $p$ for an isotropic, perfect fluid.

Finally, inserting the metric (3), the Ricci tensor and Ricci scalar as well as the energy-momentum tensor (5) into the Einstein equation $R_{\mu \nu}-1 / 2 g_{\mu \nu} R+\Lambda g_{\mu \nu}=8 \pi G_{N} / c^{4} T_{\mu \nu}$ leads to a set of four equations

$$
\begin{gathered}
\frac{c}{K f^{3} a^{2}}\left[c^{3}\left(\left(1+K \Lambda a^{2}\right) f^{3}-2 K a f^{\prime} a^{\prime}+K f\left(a^{\prime 2}+2 a^{\prime \prime}\right)\right)\right. \\
\left.-2 K a f^{3} \dot{c} \dot{a}+K c f^{3}\left(\dot{a}^{2}+2 a \ddot{a}\right)\right]=-8 \pi G_{N} p \\
-3 c^{2} \dot{a}^{\prime}=8 \pi G_{N} f^{2} \rho a \\
\frac{1}{K a^{2} f^{3}}\left[c^{2}\left(\left(3+K \Lambda a^{2}\right) f^{3}-3 K a f^{\prime} a^{\prime}+3 K f\left(a^{\prime 2}+a a^{\prime \prime}\right)\right)+3 K f^{3} \dot{a}^{2}\right]=8 \pi G_{N} \rho
\end{gathered}
$$




$$
\frac{c}{K a^{2}}\left[c^{3}\left(\left(3+K \Lambda a^{2}\right) f^{2}+3 K a^{\prime 2}\right)-3 K a f^{2} \dot{c} \dot{a}+3 K c f^{2}\left(\dot{a}^{2}+\ddot{a}\right)\right]=8 \pi G_{N} f^{4} \rho
$$

where the $\operatorname{dot}\left({ }^{\cdot}\right)$ denotes the time derivative with respect to $t$ and (') with respect to $\tau$.

In comparison to the $4 \mathrm{D}$ Friedmann equations, we now have two equations more allowing us to estimate the cosmological constant $\Lambda$ and the curvature of Universe. This is a similar approach to the original idea by Kaluza and Klein [40] [41] where they used a five-dimensional space time with four space dimensions to decouple 4D electromagnetism from 5D relativity.

\section{Discussion and Limits of the Five-Dimensional Friedmann Equations}

In this section, we are going to reduce Equations (6)-(9) and derive analytic expressions for the cosmological constant $\Lambda$ and the curvature $1 / \sqrt{|K|}$.

Combining Equations (6)-(8) leads to the conservation of energy

$$
-\left(3 \dot{a} \frac{p}{c^{2}}+3 \dot{a} \rho+a \dot{\rho}+2 \frac{\dot{c}}{c} a \rho\right)=\frac{f^{\prime}}{f} a \rho+3 a^{\prime} \rho+a \rho^{\prime}
$$

which relates the scale factor $a$, the pressure $p$ and the density $\rho$ to each other.

We divide the pressure into two terms coming from the matter and radiation in the Universe. We assume that the motion of particles in the Universe on a global scale is isotropic and collisionless, therefore the matter pressure is negligible, hence $p_{\text {mat }}=0$ Solving (10) leads to $\rho_{\text {mat }}=C_{\text {mat }} \frac{1}{a^{3} c^{2}} \frac{f\left(-t+\tau+t_{l}\right)}{f(\tau)}$ with a lower time limit $t_{l}$, and an integration constant $C_{\text {mat }}$. Since $f$ depends on $\tau$ only, $f$ needs to be constant, hence $\rho_{\text {mat }}=C_{m a t} \frac{1}{a^{3} c^{2}}$. The radiation pressure is connected to the density through $p_{\text {rad }}=\rho c^{2} / 3$ [42]. Using that $f$ is constant, the solution of the resultant differential Equation (10) gives $\rho_{\text {rad }}=C_{\text {rad }} \frac{1}{a^{4} c^{2}} \mathrm{e}^{\int_{t / t}^{t} \frac{a^{\prime}(\bar{t},-t+\tau+\bar{t})}{a(\bar{t},-t+\tau+\bar{t})}}$. The total density is thus given through

$$
\rho=C_{\text {mat }} \frac{1}{a^{3} c^{2}}+C_{r a d} \frac{1}{a^{4} c^{2}} \mathrm{e}^{\int_{t_{t}}^{t} \mathrm{~d} \frac{a^{\prime}(\bar{t},-t+\tau+\bar{t})}{a(\bar{t},-t+\tau+\bar{t})}}
$$

where the lower limit $t_{l}$ is a time close to the origin of the Universe, hence we set $t_{l}=t_{\text {Planck }} \approx 10^{-43} \mathrm{~s} \approx 0$. We will show later (20) that we can assume $a_{\tau}$ to be constant and therefore $\exp \left(\int_{t_{l}}^{t} \mathrm{~d} \bar{t} \frac{a^{\prime}(\bar{t},-t+\tau+\bar{t})}{a(\bar{t},-t+\tau+\bar{t})}\right)=1$ for all $t$. Note that the conservation of energy (10) is equivalent to the acceleration Equation (6), therefore we are left with the three Equations (7)-(9) relating the two velocities $f, c$, the curvature $\sqrt{1 /|K|}$, the cosmological constant $\Lambda$ and the scaling parameter $a$ to each other. 
Generally the scaling parameter depends on both time dimensions $t$ and $\tau$. However, we know that we only observe one time dimension together with three spatial dimensions. Because of this and as a standard method to solve differential equations with two independent variables, we here choose a separation ansatz for $a(t, \tau)$ which is usually either a product ansatz or a sum ansatz. As we will discuss below the system of equations considered here can be solved using a sum ansatz. Since the second time dimension cannot be observed at the present stage of the Universe's evolution, $a(t, \tau)$ needs to be of the form $\sim a_{t}(t)+\alpha(t) a_{\tau}(\tau)$ such that $\lim _{t \rightarrow \infty} \alpha(t)=0$. Therefore, the second time dimension can only operate on a small spatial scale which is not accessible to experiments directly. Therefore, we make the ansatz

$$
a(t, \tau)=a_{t}(t)+\mathrm{e}^{\beta t} a_{\tau}(\tau), \beta<0
$$

with a contribution $a_{t}(t)$ for the first time dimension only and a contribution $\mathrm{e}^{\beta t} a_{\tau}(\tau)$ for the second time dimension such that $a(t, \tau) \rightarrow a_{t}(t)$ for large $t$. This implies that the second time dimension expands the Universe by a small value $a_{\tau}$ not measurable with current methods. Note that in theory, there is a whole class of functions $\alpha(t)$ satisfying $\lim _{t \rightarrow \infty} \alpha(t)=0$; however, since Equation (12) needs to be inserted into a system of differential equations, we require a function which is sufficiently smooth and differentiable, which is why we choose the exponential function. On the other hand, we assume that $a_{t}(t)$ tends to 0 for $t \rightarrow 0$ which is the usual assumption for the Big Bang. Yet, unlike a universe with four space-time dimensions, the overall scaling parameter $a(t, \tau)$ tends to $a_{\tau}(\tau) \neq 0$ for $t \rightarrow 0$; hence we avoid a singularity for $t \rightarrow 0$ [43] [44] [45] [46].

For the speed of light, we make the ansatz

$$
c(t)=c_{0}+\frac{\tilde{c}}{t-t_{0}}
$$

since the speed of light is time-dependent with $t^{-1}$ for small $t$ and becomes $c_{0} \approx 3 \times 10^{8} \mathrm{~m} \cdot \mathrm{s}^{-1}$ for $t \gg t_{0}$ and reference length and time $\tilde{c}$ and $t_{0}$ [36] [37].

Inserting (12) and (13) into (7)-(9) leads to the equations

$$
\begin{aligned}
& -3\left(c_{0}+\frac{\tilde{c}}{t-t_{0}}\right) \beta \mathrm{e}^{\beta t} a_{\tau}^{\prime} \\
& =8 \pi G_{N} f^{2}\left(a_{t}+\mathrm{e}^{\beta t} a_{\tau}\right)\left(C_{m a t} \frac{1}{\left(a_{t}+\mathrm{e}^{\beta t} a_{\tau}\right)^{3}\left(c_{0}+\frac{\tilde{c}}{t-t_{0}}\right)^{2}}\right. \\
& \left.+C_{\text {rad }} \frac{1}{\left(a_{t}+\mathrm{e}^{\beta t} a_{\tau}\right)^{4}\left(c_{0}+\frac{\tilde{c}}{t-t_{0}}\right)^{2}} \mathrm{e}^{\int^{t} \mathrm{~d} \bar{t} \frac{a^{\prime}(\bar{t},-t+\tau+\bar{t})}{a(\bar{t},-t+\tau+\bar{t})}}\right)
\end{aligned}
$$




$$
\begin{aligned}
& \frac{1}{K\left(a_{t}+\mathrm{e}^{\beta t} a_{\tau}\right)^{2} f^{3}}\left[( c _ { 0 } + \frac { \tilde { c } } { t - t _ { 0 } } ) ^ { 2 } \left(\left(3+K \Lambda\left(a_{t}+\mathrm{e}^{\beta t} a_{\tau}\right)^{2}\right) f^{3}\right.\right. \\
& \left.\left.+3 K f\left(\mathrm{e}^{2 \beta t} a_{\tau}^{\prime 2}+\left(a_{t}+\mathrm{e}^{\beta t} a_{\tau}\right) \mathrm{e}^{\beta t} a_{\tau}^{\prime \prime}\right)\right)+3 K f^{3}\left(\dot{a}_{t}+\beta \mathrm{e}^{\beta t} a_{\tau}\right)^{2}\right] \\
& =8 \pi G_{N}\left(C_{m a t} \frac{1}{\left(a_{t}+\mathrm{e}^{\beta t} a_{\tau}\right)^{3}\left(c_{0}+\frac{\tilde{c}}{t-t_{0}}\right)^{2}}\right. \\
& \left.+C_{r a d} \frac{1}{\left(a_{t}+\mathrm{e}^{\beta t} a_{\tau}\right)^{4}\left(c_{0}+\frac{\tilde{C}}{t-t_{0}}\right)^{2}} \mathrm{e}^{\int_{t}^{t} \mathrm{~d} \bar{t} \frac{a^{\prime}(\bar{s},-t+\tau+\bar{t})}{a(\bar{t},-t+\tau+\bar{t})}}\right) \\
& \frac{\left(c_{0}+\frac{\tilde{c}}{t-t_{0}}\right)}{K\left(a_{t}+\mathrm{e}^{\beta t} a_{\tau}\right)^{2}}\left[\left(c_{0}+\frac{\tilde{c}}{t-t_{0}}\right)^{3}\left(\left(3+K \Lambda\left(a_{t}+\mathrm{e}^{\beta t} a_{\tau}\right)^{2}\right) f^{2}+3 K \mathrm{e}^{\beta t} a_{\tau}^{\prime 2}\right)\right. \\
& -3 K\left(a_{t}+\mathrm{e}^{\beta t} a_{\tau}\right) f^{2}\left(-\frac{\tilde{c}}{\left(t-t_{0}\right)^{2}}\right)\left(\dot{a}_{t}+\beta \mathrm{e}^{\beta t} a_{\tau}\right) \\
& \left.+3 K\left(c_{0}+\frac{\tilde{c}}{t-t_{0}}\right) f^{2}\left(\left(\dot{a}_{t}+\beta \mathrm{e}^{\beta t} a_{\tau}\right)^{2}+\left(\ddot{a}_{t}+\beta^{2} \mathrm{e}^{\beta t} a_{\tau}\right)\right)\right] \\
& =8 \pi G_{N} f^{4}\left(C_{m a t} \frac{1}{\left(a_{t}+\mathrm{e}^{\beta t} a_{\tau}\right)^{3}\left(c_{0}+\frac{\tilde{c}}{t-t_{0}}\right)^{2}}\right. \\
& \left.+C_{r a d} \frac{1}{\left(a_{t}+\mathrm{e}^{\beta t} a_{\tau}\right)^{4}\left(c_{0}+\frac{\tilde{C}}{t-t_{0}}\right)^{2}} \mathrm{e}^{\int^{t} \mathrm{~d} \bar{t} \frac{a^{\prime}(\bar{t},-t+\tau+\bar{t})}{a(\bar{t},-t+\tau+\bar{t})}}\right) .
\end{aligned}
$$

We now use this system of equations to derive analytic expressions for the cosmological constant and the Universe's curvature. But before doing so, we discuss this system for very large and very small $t$.

Since $\rho \sim a_{t}^{-3}+a_{t}^{-4}$ Equation (14) for large $t$ implies that the scaling factor and hence the Universe are growing. For $t \rightarrow \infty$ and identifying $f=c_{0}$ (15) and (16) simply become the Friedmann equation for four space-time dimensions [44] [45].

For small $t, t \rightarrow 0$, and using $f=c_{0}$, we get

$$
-3\left(c_{0}-\frac{\tilde{c}}{t_{0}}\right)^{2} \beta a_{\tau}^{\prime}=8 \pi G_{N} c_{0}^{2}\left(C_{\text {mat }} \frac{1}{a_{\tau}^{2}\left(c_{0}-\frac{\tilde{c}}{t_{0}}\right)^{2}}+C_{r a d} \frac{1}{a_{\tau}^{3}\left(c_{0}-\frac{\tilde{c}}{t_{0}}\right)^{2}}\right)
$$




$$
\begin{aligned}
& \frac{3\left(c_{0}-\frac{\tilde{c}}{t_{0}}\right)^{2}}{K a_{\tau}^{2}}+\Lambda\left(c_{0}-\frac{\tilde{c}}{t_{0}}\right)^{2}+\frac{3\left(c_{0}-\frac{\tilde{c}}{t_{0}}\right)^{2}}{c_{0}^{2} a_{\tau}^{2}}\left(a_{\tau}^{\prime 2}+a_{\tau} a_{\tau}^{\prime \prime}\right)+\frac{3\left(\dot{a}_{t}(t=0)+\beta a_{\tau}\right)^{2}}{a_{\tau}^{2}} \\
= & 8 \pi G_{N}\left(C_{\text {mat }} \frac{1}{a_{\tau}^{3}\left(c_{0}-\frac{\tilde{c}}{t_{0}}\right)^{2}}+C_{\text {rad }} \frac{1}{a_{\tau}^{4}\left(c_{0}-\frac{\tilde{c}}{t_{0}}\right)^{2}}\right) \\
& \frac{c_{0}-\frac{\tilde{c}}{t_{0}}}{K a_{\tau}^{2}}\left[\left(c_{0}-\frac{\tilde{c}}{t_{0}}\right)^{3}\left(\left(3+K \Lambda a_{\tau}^{2}\right) c_{0}^{2}+3 K a_{\tau}^{\prime 2}\right)+3 K c_{0}^{2} \frac{\tilde{c}}{t_{0}^{2}} a_{\tau}\left(\dot{a}_{t}(t=0)+\beta a_{\tau}\right)\right. \\
& \left.+3 K c_{0}^{2}\left(c_{0}-\frac{\tilde{c}}{t_{0}}\right)\left(\left(\dot{a}_{t}(t=0)+\beta a_{\tau}\right)^{2}+a_{\tau}\left(\ddot{a}_{t}(t=0)+\beta^{2} a_{\tau}\right)\right)\right] \\
= & 8 \pi G_{N} c_{0}^{4}\left(C_{\text {mat }} \frac{1}{a_{\tau}^{3} c^{2}}+C_{r a d} \frac{1}{a_{\tau}^{4} c^{2}}\right) .
\end{aligned}
$$

In the following, we estimate the values of $a_{\tau}$, of $K$ and of $\Lambda$. Since we do not know the exact values for the density $\rho$ as well as for the constants $t_{0}, \tilde{C}$ and $\beta$, we here limit ourselves to give ranges for $K$ and $\Lambda$. Furthermore, we use $G_{N} \approx 6.674 \times 10^{-11} \mathrm{~m}^{3} \cdot \mathrm{kg}^{-1} \cdot \mathrm{s}^{-2}$ and $c_{0} \approx 3 \times 10^{8} \mathrm{~m} \cdot \mathrm{s}^{-1}$ for the gravitational constant and the speed of light. In standard cosmology [47], the ratios $\Omega_{\text {mat }}$ and $\Omega_{\text {rad }}$ of matter and radiation in our Universe are connected to the density (11) and hence to $a$ and $c_{0}$ through

$$
C_{\text {mat }} \approx \frac{3 H_{0}^{2}}{8 \pi G} \Omega_{\text {mat }} a^{3} c_{0}^{2} \text { and } C_{\text {rad }} \approx \frac{3 H_{0}^{2}}{8 \pi G} \Omega_{r a d} a^{4} c_{0}^{2}
$$

where we use $H_{0} \approx 70 \mathrm{~km} \cdot \mathrm{s}^{-1} \cdot(\mathrm{Mpc})^{-1} \approx 2.27 \times 10^{-18} \mathrm{~s}^{-1}$ [48]. Note that for small $t a$ tends to $a_{\tau}$ and that $a=\ell / R_{o b s}$ is the scaling parameter which relates the actual size $\ell$ of the Universe at times $(t, \tau)$ to the size $R_{\text {obs }} \approx 46.6 \mathrm{GLy} \approx 4.41 \times 10^{26} \mathrm{~m}$ of the observable Universe.

We finally need to give estimates for $\tilde{c}, t_{0}$ and $\beta$ before we can continue our discussion. Since we know that the contribution of $a_{\tau}$ to the whole scaling factor $a(t, \tau)$ (12) needs to vanish not only for $t \rightarrow \infty$, but also for the observable Universe, we require $\exp \left(\beta t_{\text {obs }}\right) \ll 1$ for $t_{\text {obs }} \approx 13.8 \mathrm{Ga} \approx 4.35 \times 10^{17} \mathrm{~s}$, and hence $\beta \ll \log (1.0) / t_{o b s} \approx 0 \mathrm{~s}^{-1}$; we here choose $\beta \ll\left(-10^{-5}\right)-\left(-10^{-15}\right) \mathrm{s}^{-1}$ such that $\exp \left(\beta t_{\text {obs }}\right) \approx 0$. Although the speed of light might not have been constant in the Early Universe [37], it needs to be constant for the Universe today. We therefore choose $t_{0}=t_{\text {Planck }} \approx 10^{-43} \mathrm{~s}$ to be on the Planck scale as well as $\tilde{c} /\left(t_{o b s}-t_{0}\right) \ll 1 \mathrm{~m} \cdot \mathrm{s}^{-1}$ or equivalently $\tilde{c} \ll\left(t_{o b s}-t_{0}\right) \mathrm{m} \cdot \mathrm{s}^{-1} \approx 4.35 \times 10^{17} \mathrm{~m}$.

For these estimates, Figure 1 shows $a_{\tau}^{\prime}(\tau) / a_{\tau}(\tau)$ as a function of $a_{\tau}(\tau)$ (following from Equation (17) for various $\Omega_{\text {mat }}$ and $\Omega_{\text {rad }}$. It illustrates that the growth of $a_{\tau}$ is negligible relative to its size; therefore we assume $a_{\tau}$ to be constant for all $\tau$, hence $a_{\tau}^{\prime} \equiv 0$. Thus, retrospectively, we see indeed that the integral in (11) disappears. Since we have no direct measurements of the second time dimension $\tau, a_{\tau}$ needs to be small; from previous work [36], we know 


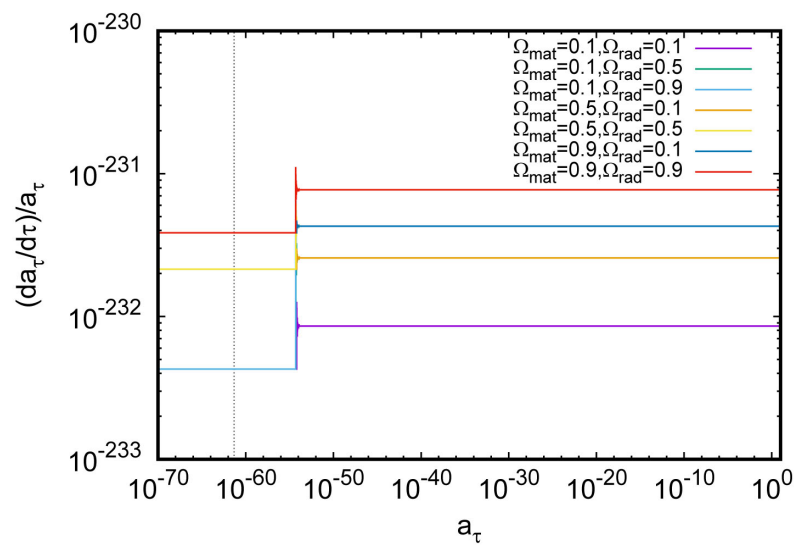

Figure 1. $a_{\tau}^{\prime}(\tau) / a_{\tau}(\tau)$ as a function of $a_{\tau}(\tau)$ for fixed $\beta=-10^{-10} \mathrm{~s}^{-1}, \tilde{c}=10^{17} \mathrm{~m}$ and various $\Omega_{\text {mat }}$ and $\Omega_{\text {rad }}$. The dotted line indicates the scaling parameter for the Planck length $\ell_{\text {Planck }} \approx 10^{-35} \mathrm{~m}$.

that a second time dimension is responsible for the Planck length. Therefore, for the following, we set

$$
a_{\tau}(\tau) \approx 4.59 \times 10^{-62}=\text { const. }
$$

which is the approximate scale factor for the Planck length $\approx 10^{-35} \mathrm{~m}$.

\section{The Universe's Curvature and the Value of the Cosmological Constant}

Assuming $a_{\tau}$ to be constant, hence $a_{\tau}^{\prime}=a_{\tau}^{\prime \prime} \approx 0$, we use the remaining Equations (18) and (19) to determine $\Lambda$ and $K$ for which we still need to approximate $\dot{a}_{t}(t=0)$ and $\ddot{a}_{t}(t=0)$. We are here stuck with a dilemma since these derivatives couple the two time dimensions to each other, and there is no way to determine $\dot{a}_{t}(t=0)$ and $\ddot{a}_{t}(t=0)$ in a self-consistent way. For small $t \approx t_{\text {Planck }} \approx 0$, we are thus limited to use the four-dimensional Friedmann equation [44] [45] yielding $\dot{a}=\sqrt{\frac{8 \pi G_{N}}{3}\left(C_{m a t} \frac{1}{a_{t} c_{0}^{2}}+C_{r a d} \frac{1}{a_{t}^{2} c_{0}^{2}}\right)-\frac{c_{0}^{2}}{K}-\frac{\Lambda c_{0}^{2}}{3} a_{t}^{2}}$ and $\ddot{a}_{t}=\frac{4 \pi G_{N}}{3}\left(C_{\text {mat }} \frac{-1}{a_{t}^{2} c_{0}^{2}}+C_{r a d} \frac{-2}{a_{t}^{3} c_{0}^{2}}\right)-\frac{\Lambda c_{0}^{2}}{3} a_{t}$.

Finally using these expressions to calculate $\dot{a}_{t}(t=0)$ and $\ddot{a}_{t}(t=0)$ and inserting them into (18) and (19) yield

$$
\begin{aligned}
& \frac{3\left(c_{0}-\frac{\tilde{c}}{t_{0}}\right)^{2}}{K a_{\tau}^{2}}+\Lambda\left(c_{0}-\frac{\tilde{c}}{t_{0}}\right)^{2}+\frac{3\left(\sqrt{\frac{8 \pi G_{N}}{3}\left(C_{m a t} \frac{1}{a_{t}\left(t_{0}\right) c_{0}^{2}}+C_{r a d} \frac{1}{a_{t}\left(t_{0}\right)^{2} c_{0}^{2}}\right)-\frac{c_{0}^{2}}{K}-\frac{\Lambda c_{0}^{2}}{3} a_{t}\left(t_{0}\right)^{2}}+\beta a_{\tau}\right)^{2}}{a_{\tau}^{2}} \\
& =8 \pi G_{N}\left(C_{\text {mat }} \frac{1}{a_{\tau}^{3}\left(c_{0}-\frac{\tilde{c}}{t_{0}}\right)^{2}}+C_{\text {rad }} \frac{1}{a_{\tau}^{4}\left(c_{0}-\frac{\tilde{c}}{t_{0}}\right)^{2}}\right)
\end{aligned}
$$




$$
\begin{aligned}
& \frac{c_{0}-\frac{\tilde{c}}{t_{0}}}{K a_{\tau}^{2}}\left[\left(c_{0}-\frac{\tilde{c}}{t_{0}}\right)^{3}\left(3+K \Lambda a_{\tau}^{2}\right) f^{2}\right. \\
& +3 K f^{2} \frac{\tilde{c}}{t_{0}^{2}} a_{\tau}\left(\sqrt{\frac{8 \pi G_{N}}{3}\left(C_{m a t} \frac{1}{a_{t}\left(t_{0}\right) c_{0}^{2}}+C_{r a d} \frac{1}{a_{t}\left(t_{0}\right)^{2} c_{0}^{2}}\right)-\frac{c_{0}^{2}}{K}-\frac{\Lambda c_{0}^{2}}{3} a_{t}\left(t_{0}\right)^{2}+\beta a_{\tau}}\right) \\
& +3 K f^{2}\left(c_{0}-\frac{\tilde{c}}{t_{0}}\right)\left(\sqrt{\left.\frac{8 \pi G_{N}}{3}\left(C_{m a t} \frac{1}{a_{t}\left(t_{0}\right) c_{0}^{2}}+C_{r a d} \frac{1}{a_{t}\left(t_{0}\right)^{2} c_{0}^{2}}\right)-\frac{c_{0}^{2}}{K}-\frac{\Lambda c_{0}^{2}}{3} a_{t}\left(t_{0}\right)^{2}+\beta a_{\tau}\right)^{2}}\right. \\
& \left.\left.+a_{\tau}\left(\frac{4 \pi G_{N}}{3}\left(C_{m a t} \frac{-1}{a_{t}^{2} c_{0}^{2}}+C_{r a d} \frac{-2}{a_{t}^{3} c_{0}^{2}}\right)-\frac{\Lambda c_{0}^{2}}{3} a_{t}+\beta^{2} a_{\tau}\right)\right)\right] \\
& =8 \pi G_{N} f^{4}\left(C_{m a t} \frac{a_{\tau}^{3}\left(c_{0}-\frac{\tilde{c}}{t_{0}}\right)^{2}}{a_{\tau}^{4}\left(c_{0}-\frac{\tilde{c}}{t_{0}}\right)^{2}}\right) .
\end{aligned}
$$

These two equations give two sets of solutions $\left(\Lambda_{1}, K_{1}\right)$ and $\left(\Lambda_{2}, K_{2}\right)$ which are very lengthy and which we have therefore added to the supplementary Mathematica script.

Figure 2 shows the solution $\left(\Lambda_{1}, K_{1}\right)$ for various $\Omega, \beta$ and $\tilde{C}$; note that the solutions $\left(\Lambda_{1}, K_{1}\right)$ and $\left(\Lambda_{2}, K_{2}\right)$ give similar values. Figure $2(a)$ and Figure 2(b) shows that for a given set of $\beta$ and $\tilde{c}, \Lambda$ and $|K|$ are almost constant and only vary slightly for different $\Omega_{\text {rad }}$ and $\Omega_{\text {mat }}$. In contrast, the dependence on $\beta$ and $\tilde{C}$ is much more significant with bridging several orders of magnitude. Hence, $|K|$ varies from approx. $10^{174} \mathrm{~m}^{2}$ to $10^{216} \mathrm{~m}^{2}$ for all considered cases which implies that the curvature $\sqrt{1 /|K|}$ is approximately 0 . Additionally, $\Lambda$ varies between $10^{-90} \mathrm{~m}^{-2}$ and $10^{-51} \mathrm{~m}^{-2}$ which is well in agreement with measurements determining $\Lambda$ to approx. $10^{-52} \mathrm{~m}^{-2}$ [11]. In addition, we have performed parameter studies how $\Lambda$ and $K$ depend on $a_{\tau}, a_{t}\left(t_{0}\right)$ and $t_{0}$ for fixed $\beta=-10^{-10} \mathrm{~s}^{-1}, \tilde{c}=10^{10} \mathrm{~m}, \Omega_{\text {mat }}=0.5$ and $\Omega_{\text {rad }}=0.5$. For all considered cases, we observe that there is not a significant deviation from the values of $\Lambda$ and $|K|$ as shown in Figure 2, therefore not altering our conclusions.

\section{Conclusions and Outlook}

Extending the Friedmann equations by one time dimension allows us to solve several cosmological mysteries simultaneously:

1) The second time dimension leads to a small expansion of the Universe which we interpret as Planck length.

2) This small extension exists for all times $t$, including the limit $t \rightarrow 0$. Therefore, the size of the Universe does not shrink to zero for small $t$ avoiding a singularity of infinite mass and energy density at the Big Bang. 


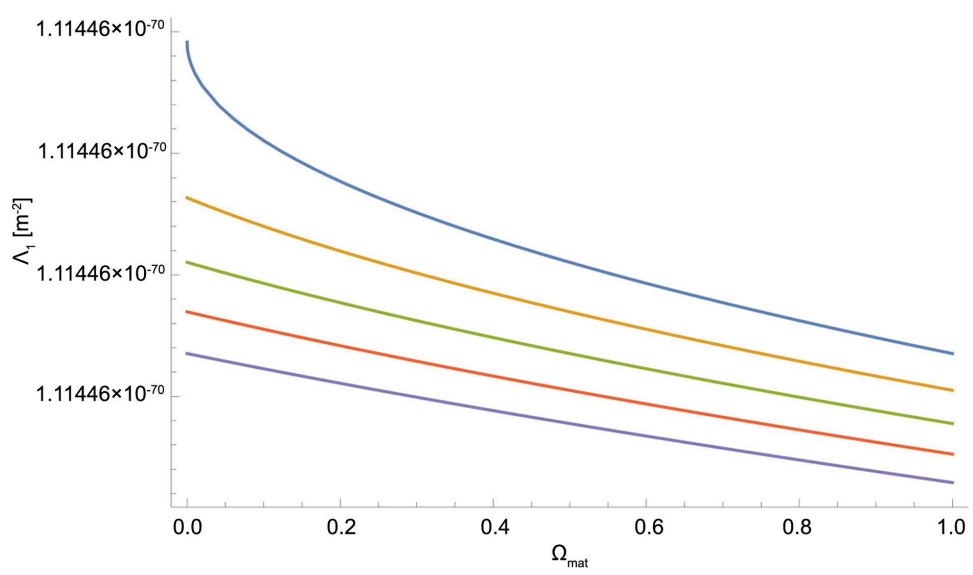

(a)

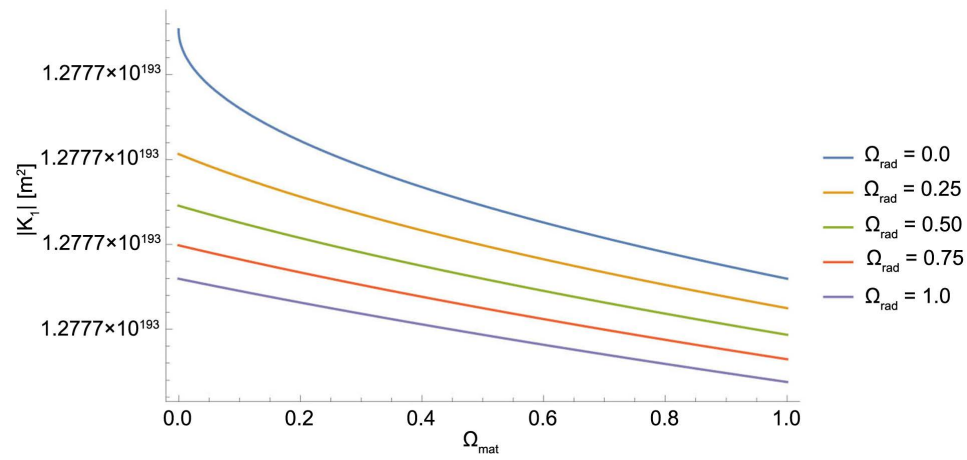

(b)

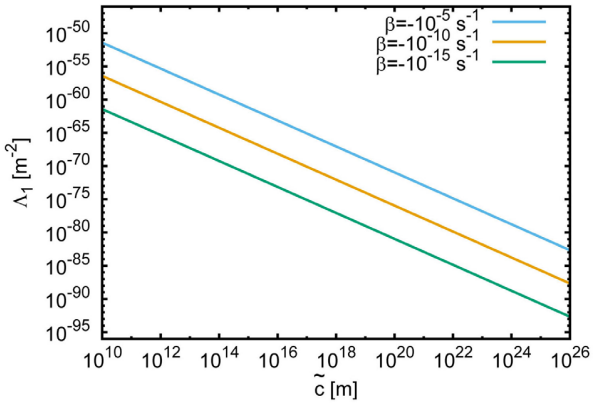

(c)

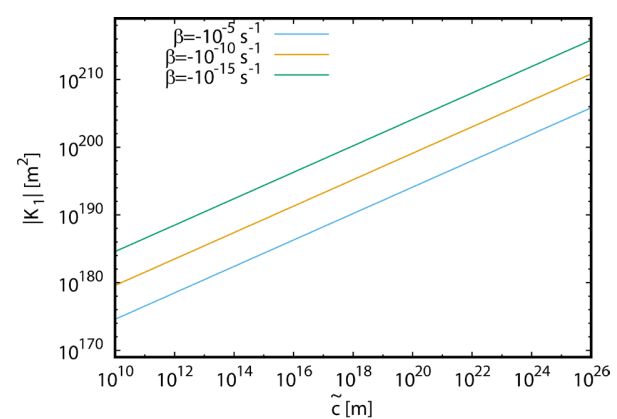

(d)

Figure 2. (a), (b) $\Lambda_{1}$ and $\left|K_{1}\right|$ as a function of $\Omega_{\text {mat }}$ for fixed $\beta=-10^{-10} \mathrm{~s}^{-1}$, $\tilde{c}=10^{17} \mathrm{~m}$ and various $\Omega_{\text {rad }}$. (c), (d) $\Lambda_{1}$ and $\left|K_{1}\right|$ as a function of $\tilde{c}$ for fixed $\Omega_{\text {mat }}=\Omega_{\text {rad }}=0.5$ and various $\beta$. 
3) The Universe's curvature is almost zero; hence the Universe is flat.

4) The cosmological constant varies between $10^{-90} \mathrm{~m}^{-2}$ and $10^{-52} \mathrm{~m}^{-2}$ which agrees with measurements [11].

The overall scenario is thus that the second time dimension expands the Universe on a very small length scale becoming manifest as the Planck length. Subsequently, from this small length scale, the Universe's curvature and the size of the cosmological constant follow. Note that the value of the cosmological constant is sometimes related to the zero-point-energy or vacuum-energy in quantum physics. However, in contrast to a quantum theory approach, we have presented a mechanism, which allows to estimate the value of the cosmological constant self-consistently from the Friedmann equations in a $(2,3)$ space time geometry, and whilst quantum theories cannot predict the value of the cosmological constant correctly [20], we relate it to the small Planck length and are indeed capable of confining its value within a range which is consistent with measurements and thus offers a solution to the cosmological constant problem. Since the cosmological constant, equivalent to the thermodynamic pressure, might be related to the effect of gravity's rainbow in the vicinity of black holes [49] [50] [51] [52] [53], this poses the question whether a second time dimension is really the fundamental property of space-time for the sensitivity of photons on gravity depending on their wavelengths. However, this is out of the scope of this paper and will be subject to future research.

Furthermore, it is interesting to note that we have not presumed any periodicity or compactness of the second time dimension as normally assumed for additional space dimensions. The effect of the second time dimension, however, is limited to a spatial scale of the size of the Planck length only. Therefore, the second time dimension does not act on the macroscopic Universe and is thus not visible to current experiments so far. However, this does not exclude that the proposed scenario can be tested in the future with advanced technology.

The presented approach gives novel ideas on how to approach cosmological singularities in the future. The connection of vanishing singularities and additional time dimensions gives rise to investigate the Big Bang more thoroughly in the context of extended space-time. Beyond that, we suggest to extend other metrics with additional time dimensions, such as the Schwarzschild metric or the Eddington-Finkelstein metric to study the effect of additional time dimensions on black holes.

\section{Acknowledgements}

I would like to thank Henrik Svensmark, Sara Svendsen and Khaled Alizai for careful reading and useful suggestions. The supplementary material for this publication can be found at 10.5281/zenodo.4007757.

\section{Conflicts of Interest}

The author declares no conflicts of interest regarding the publication of this paper. 


\section{References}

[1] Einstein, A. (1917) Kosmologische Betrachtungen zur allgemeinen Relativitätstheorie. In: Sitzungsberichte der Königlich Preußischen Akademie der Wissenschaften, Verlag Der Bayer Akad D Wiss, Berlin, 142-152.

[2] Zwicky, F. (1933) Die Rotverschiebung von extragalaktischen Nebeln. Helvetica Physica Acta, 6, 110-127.

[3] Riess, A.G., Filippenko, A.V., Challis, P., Clocchiatti, A., Diercks, A., Garnavich, P.M., Gilliland, R.L., Hogan, C.J., Jha, S. and Kirshner, R.P. (1998) Observational Evidence from Supernovae for an Accelerating Universe and a Cosmological Constant. The Astronomical Journal, 116, 1009. https://doi.org/10.1086/300499

[4] Perlmutter, S., Aldering, G., Goldhaber, G., Knop, R.A., Nugent, P., Castro, P.G., Deustual, S., Fabbro, S., Goobar, A. and Groom, D.E. (1999) Measurements of $\Omega$ and $\Lambda$ from 42 High-Redshift Supernovae. The Astrophysical Journal, 517, 565-586. https://doi.org/10.1086/307221

[5] Huterer, D. and Turner, M.S. (1999) Prospects for Probing the Dark Energy via Supernova Distance Measurements. Physical Review D, 60, Article ID: 081301. https://doi.org/10.1103/PhysRevD.60.081301

[6] Clowe, D., Bradac, M., Gonzalez, A.H., Markevitch, M., Randall, S.W., Jones, C. and Zaritsky, D. (2006) A Direct Empirical Proof of the Existence of Dark Matter. The Astrophysical Journal, 648, L109. https://doi.org/10.1086/508162

[7] Spergel, D.N. (2015) The Dark Side of the Cosmology: Dark Matter and Dark Energy. Science, 347, 1100-1102. https://doi.org/10.1126/science.aaa0980

[8] Efstathiou, G. and Bond, J.R. (1999) Cosmic Confusion: Degeneracies among Cosmological Parameters Derived from Measurements of Microwave Background Anisotropies. Monthly Notices of the Royal Astronomical Society, 304, 75-79. https://doi.org/10.1046/j.1365-8711.1999.02274.x

[9] Knox, L., Christensen, N. and Skordis, C. (2001) The Age of the Universe and the Cosmological Constant Determined from Cosmic Microwave Background Anisotropy Measurements. The Astrophysical Journal Letters, 563, L95-L98. https://doi.org/10.1086/338655

[10] Netterfield, C.B., et al. (2001) A Measurement by Boomerang of Multiple Peaks in the Angular Power Spectrum of the Cosmic Microwave Background. The Astrophysical Journal, 571, 604. https://doi.org/10.1086/340118

[11] The Planck Collaboration (2018) Planck 2018 Results. VI. Cosmological Parameters.

[12] Hameeda, M., Upadhyay, S., Faizal, M. and Ali, A.F. (2016) Effects of Cosmological Constant on Clustering of Galaxies. Monthly Notices of the Royal Astronomical Society, 463, 3699-3704. https://doi.org/10.1093/mnras/stw2202

[13] Magueijo, J. and Smolin, L. (2004) Gravity's Rainbow. Classical Quantum Gravity, 21, 1725-1736. https://doi.org/10.1088/0264-9381/21/7/001

[14] Hendi, S.H., Panahiyan, S., Panah, B.E., Faizal, M. and Momennia, M. (2016) Critical Behavior of Charged Black Holes in Gauss-Bonnet Gravity's Rainbow. Physical Review D, 94, Article ID: 024028. https://doi.org/10.1103/PhysRevD.94.024028

[15] Hendi, S.H., Faizal, M., Panah, B.E. and Panahiyan, S. (2016) Charged Dilatonic Black Holes in Gravity's Rainbow. The European Physical Journal C, 76, Article No. 296. https://doi.org/10.1140/epjc/s10052-016-4119-4

[16] Momeni, D., Faizal, M., Myrzakulov, K. and Myrzakulov, R. (2017) Fidelity Susceptibility as Holographic PV-Criticality. Physics Letters B, 765, 154-158.

https://doi.org/10.1016/j.physletb.2016.12.006 
[17] Zel'dovich, Y.B. (1967) The Cosmological Constant and Elementary Particles. JETP Letters, 6, 316-317.

[18] Zel'dovich, Y.B. (1968) The Cosmological Constant and the Theory of Elementary Particles. Soviet Physics Uspekhi, 11, 381-393. https://doi.org/10.1070/PU1968v011n03ABEH003927

[19] Abbott, L. (1988) The Mystery of the Cosmological Constant. Scientific American, 258, 106-113. https://doi.org/10.1038/scientificamerican0588-106

[20] Weinberg, S. (1989) The Cosmological Constant Problem. Reviews of Modern Physics, 61, 1-23. https://doi.org/10.1103/RevModPhys.61.1

[21] Corda, C. (2009) Interferometric Detection of Gravitational Waves: The Definitive test for General Relativity. International Journal of Modern Physics D, 18, 2275-2282.

[22] Marcolli, M. and Pierpaoli, E. (1997) Early Universe Models from Noncommutative Geometry. Advances in Theoretical and Mathematical Physics, 14, 1373-1432. https://doi.org/10.4310/ATMP.2010.v14.n5.a2

[23] Kruglov, S.I. and Faizal, M. (2015) Wave Function of the Universe from a Matrix-Valued First-Order Formalism. International Journal of Geometric Methods in Modern Physics, 12, Article ID: 1550050. https://doi.org/10.1142/S0219887815500504

[24] Garattini, R. and Faizal, M. (2016) Cosmological Constant from a Deformation of the Wheeler-DeWitt Equation. Nuclear Physics B, 905, 313-326. https://doi.org/10.1016/j.nuclphysb.2016.02.023

[25] Bars, I. (1996) Supersymmetry, $P$-Brane Duality and Hidden Space-Time Dimensions. Physical Review D, 54, 5203-5210. https://doi.org/10.1103/PhysRevD.54.5203

[26] Bars, I. and Kounnas, C. (1997) Theories with Two Times. Physics Letters B, 402, 25-32. https://doi.org/10.1016/S0370-2693(97)00452-8

[27] Bars, I. and Kounnas, C. (1997) String and Particles with Two Times. Physical Review $D$, 56, 3664-3671. https://doi.org/10.1103/PhysRevD.56.3664

[28] Araya, I.J. and Bars, I. (2014) Generalized Dualities in One-Time Physics as Holographic Predictions from Two-Time Physics. Physical Review D, 89, Article ID: 066011. https://doi.org/10.1103/PhysRevD.89.066011

[29] Chen, X. (2005) Three Dimensional Time Theory: To Unify the Principles of Basic Quantum Physics and Relativity.

[30] Bars, I. and Rey, S.-J. (2001) Noncommutative $\operatorname{Sp}(2, R)$ Gauge Theories: A field theory Approach to Two-Time Physics. Physical Review D, 64, Article ID: 046005. https://doi.org/10.1103/PhysRevD.64.046005

[31] Bars, I. and Kuo, Y.-C. (2006) Interacting Two-Time Physics Field Theory with a BRST Gauge Invariant Action. Physical Review D, 74, Article ID: 085020. https://doi.org/10.1103/PhysRevD.74.085020

[32] Romero, J.M. and Zamora, A. (2004) Snyder Noncommutative Space-Time from Two-Time Physics. Physical Review D, 70, Article ID: 105006. https://doi.org/10.1103/PhysRevD.70.105006

[33] Carrisi, M.C. and Mignemi, S. (2010) Snyder-De Sitter Model from Two-Time Physics. Physical Review D, 82, Article ID: 105031.

https://doi.org/10.1103/PhysRevD.82.105031

[34] Tegmark, M. (1997) On the Dimensionality of Spacetime. Classical and Quantum Gravity, 17, L69-L75. https://doi.org/10.1088/0264-9381/14/4/002 
[35] Piceno, E., Rosado, A. and Sadurní, E. (2016) Fundamental Constraints on Two-Time Physics. The European Physical Journal Plus, 131, Article No. 352. https://doi.org/10.1140/epjp/i2016-16352-7

[36] Köhn, C. (2017) The Planck Length and the Constancy of the Speed of Light in Five Dimensional Spacetime Parametrized with Two Time Coordinates. Journal of High Energy Physics, Gravitation and Cosmology, 3, 635-650. https://doi.org/10.4236/jhepgc.2017.34048

[37] Albrecht, A. and Magueijo, J. (1999) A Time Varying Speed of Light as a Solution to Cosmological Puzzles. Physical Review D, 59, Article ID: 043516. https://doi.org/10.1103/PhysRevD.59.043516

[38] Lemaître, G. (1931) A Homogeneous Universe of Constant Mass and Increasing Radius Accounting for the Radial Velocity of Extra-Galactic Nebulae. General Relativity and Gravitation, 45, 1635-1646.

[39] Robertson, H.P. (1935) Kinematics and World Structure. Astrophysical Journal, 82, 284. https://doi.org/10.1086/143681

[40] Kaluza, T. (1921) Zum Unitätsproblem in der Physik, Sitzungsber. Preuss. Akad. Wiss., Berlin, 966.

[41] Klein, O. (1926) Quantentheorie und fünfdimensionale Relativitätstheorie. Zeitschrift für Physik, 37, 895-906. https://doi.org/10.1007/BF01397481

[42] Landau L.D. and Lifshitz, E.M. (1971) The Classical Theory of Fields. Pergamon Press, Oxford.

[43] Hubble, E. (1929) A Relation between Distance and Radial Velocity among extra-Galactic Nebulae. Proceedings of the National Academy of Sciences of the United States of America, 15, 168-173. https://doi.org/10.1073/pnas.15.3.168

[44] Friedmann, A. (1922) Über die Krümmung des Raumes. Zeitschrift für Physik, 10, 377-386. https://doi.org/10.1007/BF01332580

[45] Friedmann, A. (1924) Über die Möglichkeit einer Welt mit konstanter negativer Krümmung des Raumes. Zeitschrift für Physik, 21, 326-322.

https://doi.org/10.1007/BF01328280

[46] Penzias, A.A. and Wilson, R.W. (1965) A Measurement of Excess Antenna Temperature at $4080 \mathrm{Mc} / \mathrm{s}$. Astrophysical Journal, 142, 419-421. https://doi.org/10.1086/148307

[47] Fließbach, T. (2012) Allgemeine Relativitätstheorie. Springer Spektrum, Berlin. https://doi.org/10.1007/978-3-8274-3032-8

[48] Jarosik, N., et al. (2011) Seven-Year Wilkinson Microwave Anisotropy Probe (WMAP) Observations: Sky Maps, Systematic Errors and Basic Results. The Astrophysical Journal, 192, 14. https://doi.org/10.1088/0067-0049/192/2/14

[49] Ali, A.F., Faizal, M. and Majumder, B. (2015) Absence of an Effective Horizon for Black Holes in Gravity's Rainbow. Europhysics Letters, 109, Article ID: 20001. https://doi.org/10.1209/0295-5075/109/20001

[50] Channuie, P. (2019) Deformed Starobinsky Model in Gravity's Rainbow. The European Physical Journal C, 79, Article No. 508. https://doi.org/10.1140/epjc/s10052-019-7031-x

[51] Gim, Y. and Gwak, B. (2019) Charged Black Hole in Gravity's Rainbow: Violation of Weak Cosmic Censorship. Physics Letters B, 794, 122-129. https://doi.org/10.1016/j.physletb.2019.05.039

[52] Mota, C.E., Santos, L.C.N., Grams, G., Da Silva, F.M. and Menezes, D.P. (2019) Combined Rastall and Rainbow Theories of Gravity with Applications to Neutron 
Stars. Physical Review D, 100, Article ID: 024043.

https://doi.org/10.1103/PhysRevD.100.024043

[53] Panahiyan, S., Hendi, S.H. and Riazi, N. (2019) $\mathrm{AdS}_{4}$ Dyonic Black Holes in Gravity's Rainbow. Nuclear Physics B, 938, 388-415.

https://doi.org/10.1016/j.nuclphysb.2018.11.019 